\title{
Development Of A Realistic Smart Information Guide Solution To Support Multi- Devices
}

\author{
Mi-JungKim ${ }^{1}$, Hyu-ChanKim*2 \\ ${ }^{1}$ Health Administration, Bucheon Univ.,Bucheon, Republic of Korea \\ ${ }^{* 2}$ Computer Multimedia, Cheju Halla Univ.,Jeju, Republic of Korea \\ lallegro@ bc.ac.kr ${ }^{1}$,khc@ chu.ac.kr ${ }^{2}$
}

Article History: Received: 11 January 2021; Accepted: 27 February 2021; Published online: 5 April 2021

\begin{abstract}
In this study, a realistic smart information guide solution to support multi-devices was developed. The upload of diverse realistic contents, as well as the support of smart devices with specific characteristics and the management of integrated contents, was made possible. Additionally, it was important to guarantee the quality of the contents (AR, VR) alongside the realistic smart information guide solution. Recently, there have been many positive responses from the expansion on and utilization of services of the fourth Industrial Revolution such as AR technologies, which include image recognition-based information services, and VR technologies, which include hands-on information services using motion recognition sensors. In the future, the development and planning of AR and VR content for visitors and tourists for locations such as museums and exhibition halls will be actively required.
\end{abstract}

Keywords: Multi-device, exhibition guide system, Exhibition, convention, realistic

\section{Introduction}

The fourth Industrial Revolution has brought about the arrival of new technologies and trends in society, culture, and science as well as the acceleration of innovation and efforts in each field. The speed of change is the quickest in the ICT sector through the rebirth of fields such as AR(augmented reality), VR(virtual reality), MR(mixed reality), AI(artificial intelligence), and BigData, as well as the emergence of new concepts and fields altogether.

As a result, smart devices are being developed that support content based on the latest technology, and such devices along the lines of smartwatches, smart toys, smart glasses, smart walls, and smart DIDs are becoming more varied and diverse. Moreover, smart devices are supplementing or even replacing smartphones through inter-device content convergence, which have thus far been at the center of ICT technology.

Exhibits such as museums and art galleries have realized this ICT(Information Communication Technology) applications to combine the humanities and social fields. The limited exhibition spaces of these exhibits are designed as smart spaces that can convey information, and they often contain story-telling contents from the traditional promotional center which allows visitors to have an increasingly available chance to interact directly in exhibition activities with all five senses through connecting an exhibition's sense of realism with a virtual world in a smart space. The meaning of a Smart Information Guidance System alludes to the use of smart devices and web/application technologies to provide such services in museums, exhibition halls, art galleries, and public relation centers[1].

It is often found that currently, exhibition guides and information guidance systems for exhibitions are mainly composed of individual systems designed for each location rather than approaching them with a more standardized solution. In addition, operating these systems is often difficult due to having to build such systems from the ground up and requiring the selection of specific operators based on the type of content and device, as well as the cost and management of each individual system. Even from the point of view of the user, there is a great deal of inconvenience in having to install separate applications and programs, which not only negatively impacts customer satisfaction, but reduces overall utilization of the services provided. Overall, new developments in system integration and operation as well as the separate costs of such systems has made them burdensome, which often limits efforts to provide better services and thus renders services useless.

Conventional mobile websites have the inherent disadvantage of needing to find and retrieve information from external points without receiving it immediately from proper sources, and applications can be expensive to produce for singular, small events. Recently, various services have been made that recognize the exact location of the user through a focus on NFC (Near Field Communication) technology[2].

Up until this point, exhibition guidance has not only suffered due to a lack of guides with professional knowledge but also due to a lack of efficacy in exhibition guidance in relation to the usage of smartphones, raising the need for the development of new exhibition service technologies for exhibition guidance[3].

Therefore, it is wholly necessary to develop and supply a system that can support various types of contents and devices required by information systems such as exhibition guidance systems and can also manage the 
integrated operation. This field of exhibition information system is required for providing content services to visitors and tourists in various forms.

In this study, we are to develop an integrated exhibition guide system that supports various multimedia contents by combining IoT technologies with devices such as smart glasses(AR glasses), transparent screens, and kiosks, all of which have recently garnered some attention.

\section{Related Research}

New types of contents are being developed based on current technologies, and in turn, new types of smart devices are being developed based on those contents; additionally, the types and forms of such smart devices are becoming more and more varied, including but not limited to smartwatches, smart toys, smart glasses, smart walls, and smart DIDs. The smartphone market, which had been at the center of ICT technology, is gradually being supplemented or replaced through cross-content device convergence.

In the current existing museum information system, multimedia contents displayed through images, text, videos, and narration using kiosks or separate terminals are the main focus. Not long after the popularization of smartphones, a location-based display guidance system using "Beacon", a Bluetooth-based wireless recognition device, had been utilized frequently, but its impact has gradually lessened over time due to inaccuracies in information, difficulties in installation and maintenance, compatibility with existing systems, and overall inconvenience in use. To compensate for this, various technologies such as GPS location tracking, QR codes, and NFC recognition are being used, but this also creates an issue in system complexity and use.

Recently, there have been many positive responses from the expansion on and utilization of services of the fourth Industrial Revolution such as AR technologies, which include image recognition-based information services, and VR technologies, which include hands-on information services using motion recognition sensors.

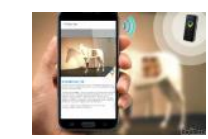

Beacon and Location Based

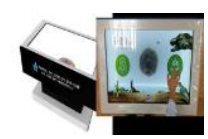

Transparent screen/ Kiosk

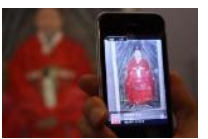

AR

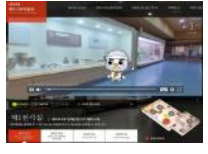

VR

$<$ Figure 1> Exhibition guidance using various technologies and devices

\subsection{Realistic content}

This is next-generation content that maximizes the utilization of the five senses based on information and communication technology(ICT) to provide a realistic experience. It is characterized by providing experience and mobility that promotes active interaction with all five senses. Virtual Reality, Augmented Reality, holograms, and five senses media are all premiere examples of realistic content, and such contents can and will be developed and applied to various fields, including not only video games and movies, but also education, health care, and automobiles[4,11].

Overall, this means that one can go beyond simply "viewing" works and exhibits and can interact with them more through the utilization of digital contents such as AR, VR, videos, and holograms through various smart devices that utilize ICT technologies.

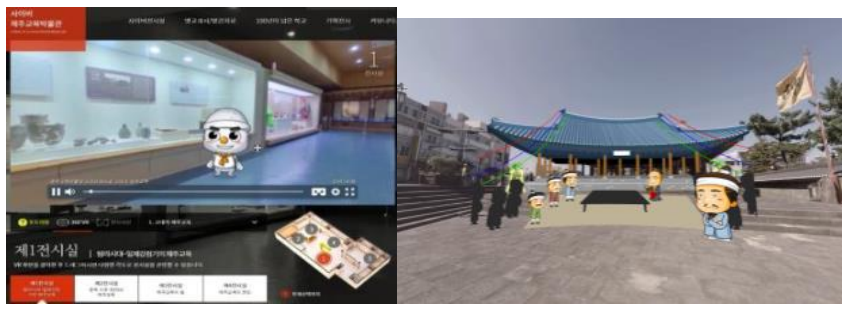

<Figure2>VR video-based character exhibition guide<Figure3> Contents based on the fusion of animation and VR

In particular, local governments are actively, increasingly producing specific experience-based exhibition contents, such as the promotion of cities and provinces, local specialties, and welfare for citizens, as well as attempts to hold hands-on exhibitions centering on local cultural heritage groups. 


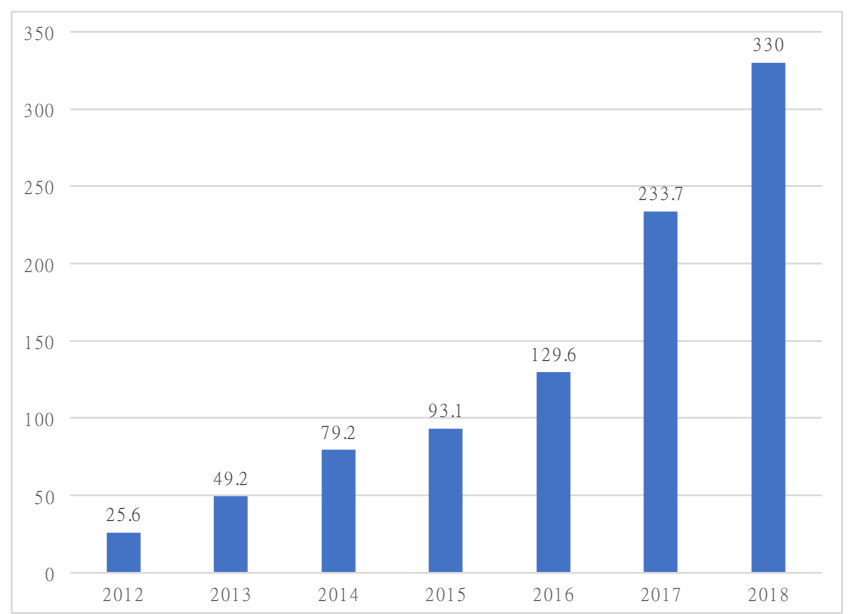

$<$ Figure 4> Ordered chart of domestic local governments' support of interactive experience centers/promotion centers(unit: 100 million KRW)

In addition to national institutions and local governments, the national government is pushing to establish experience centers in promotional facilities based on large companies by utilizing various experience-based exhibition contents technologies. In particular, when smart exhibition guidance services are applied to the domestic market, it can be seen that the number of potential applications is not insignificant. The number of registered museums includes 365 national and public museums, 754 private and university museums, 48 national and university art museums, and 190 private and university art galleries.

\subsection{Augmented Reality (AR)}

Virtual/augmented reality content technology refers to a technology that provides realistic content that allows users to undergo an experience that they are not directly experiencing in the real world due to spatial, temporal, or physical constraints through interactions that provide sensory input to the five senses, such as visual, auditory, and tactile inputs, within virtual spaces and augmented reality spaces built using computers.

Augmented Reality (AR) technology is one that synthesizes virtual objects or information onto the real world and makes them appear as though they are real objects in the real world [5,12]. In this sense, Augmented Reality (AR) technology is a key area that can revolutionize the ICT market in the future and is recognized as a potentially volatile technology that can not only greatly change the existing ICT market, but also create new markets[6].

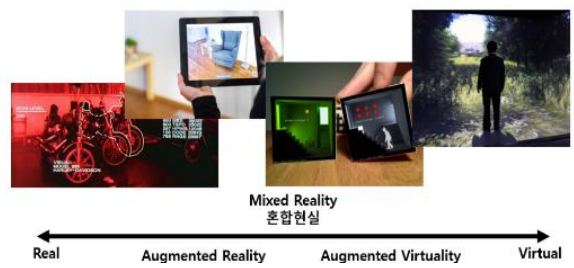

<Figure5> Virtual, Augmented, and Mixed Reality(Reality-virtual continuity) [7]

Also, museums and others use it to promote understanding and interest of visitors[8].

\subsection{AR glasses}

Smart glasses are wearable computer devices with a glasses-type frame and a transparent HMD (Head Mounted Display) function that can present images directly to the user's eyes. The market for smart glasses, a personal wearable device, is rapidly becoming a topic of discussion for the new generation information device after the smartphone [9]. After Google Glass was revealed in 2013, expectations for growth in the real-life content market, which includes AR, are increasing quickly due to the massive explosion in popularity of Pokemon Go in 2016 and the commercialization of 5G in 2019. According to a report by the market research firm Statista, the AR market is expected to grow at an average annual rate of approximately 60.3 percent from 2017 to create a global market of an estimated $\$ 198.2$ billion by 2025 [10].

The utilization of AR glasses is expanding into areas such as industrial, field, fire, and medical services, and with the emergence of various products aimed at ordinary users, the AR glasses market is expected to stabilize with a focus on the industrial AR market. 


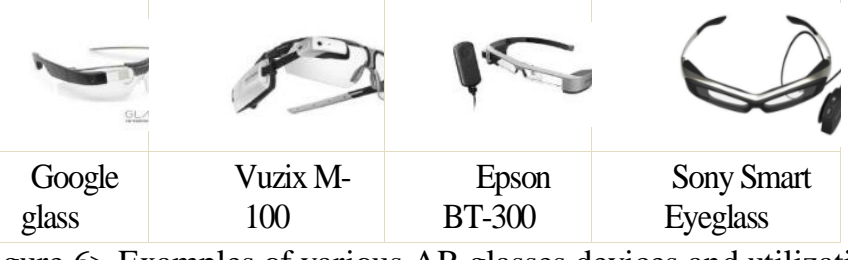

$<$ Figure 6> Examples of various AR glasses devices and utilization

Currently, smartwatches and fitness bands are leading the wearable market, but as their growth slows down, smart glasses are expected to make an entry into the mass market with a heavy focus on AR glasses.

\section{Design and Implementation}

3.1 Software and hardware composition diagram

In taking into consideration the popularization of the exhibition tourism industry, it is wholly necessary to establish a high-quality experience environment that is integrated with reality with a user-led focus that is not simply a way to convey information to said user. Therefore, it is necessary to provide realistic AR and VR content services to both domestic and foreign tourists visiting museums through contents services utilizing multidevice smart glasses, transparent screens, kiosks, and applications, and to combine such hardware with service for visitors.

The following figures show the software and hardware configurations.

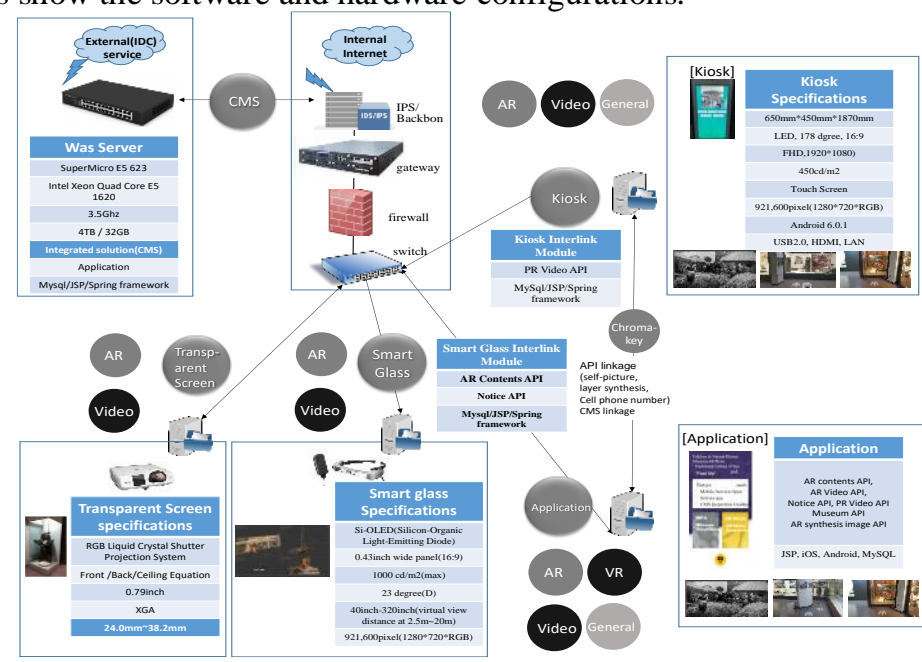

[Figure 7] Software and hardware architecture

The contents provided were developed using the Unity Engine, and various contents were presented visually to the audience by utilizing the data provided by the database.

3.2 Implementation of a service model

Smart content technologies such as AR and VR have recently expanded the base of new technologies due to rapid improvement in the quality of hardware and communication.

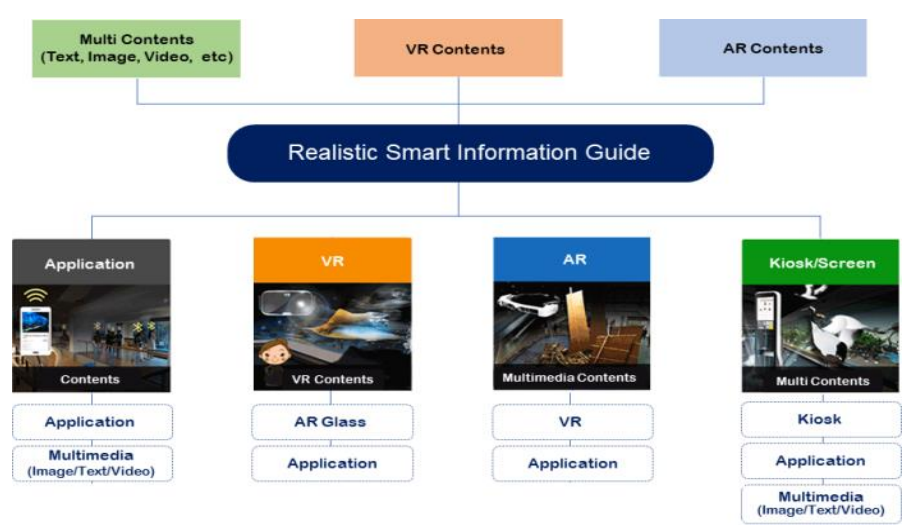

[Figure 8] Service Model Architecture 
In this study, instead of a simple experience environment using display terminals such as those commonly used in the exhibition/experience/culture/tourism industries, the implementation of a more interactive exhibition that combined actual exhibits with the surrounding environment through realistic technology was pursued.

\subsection{Implementation result}

\subsubsection{Login screen}

Log in through the admin screen and the user screen.

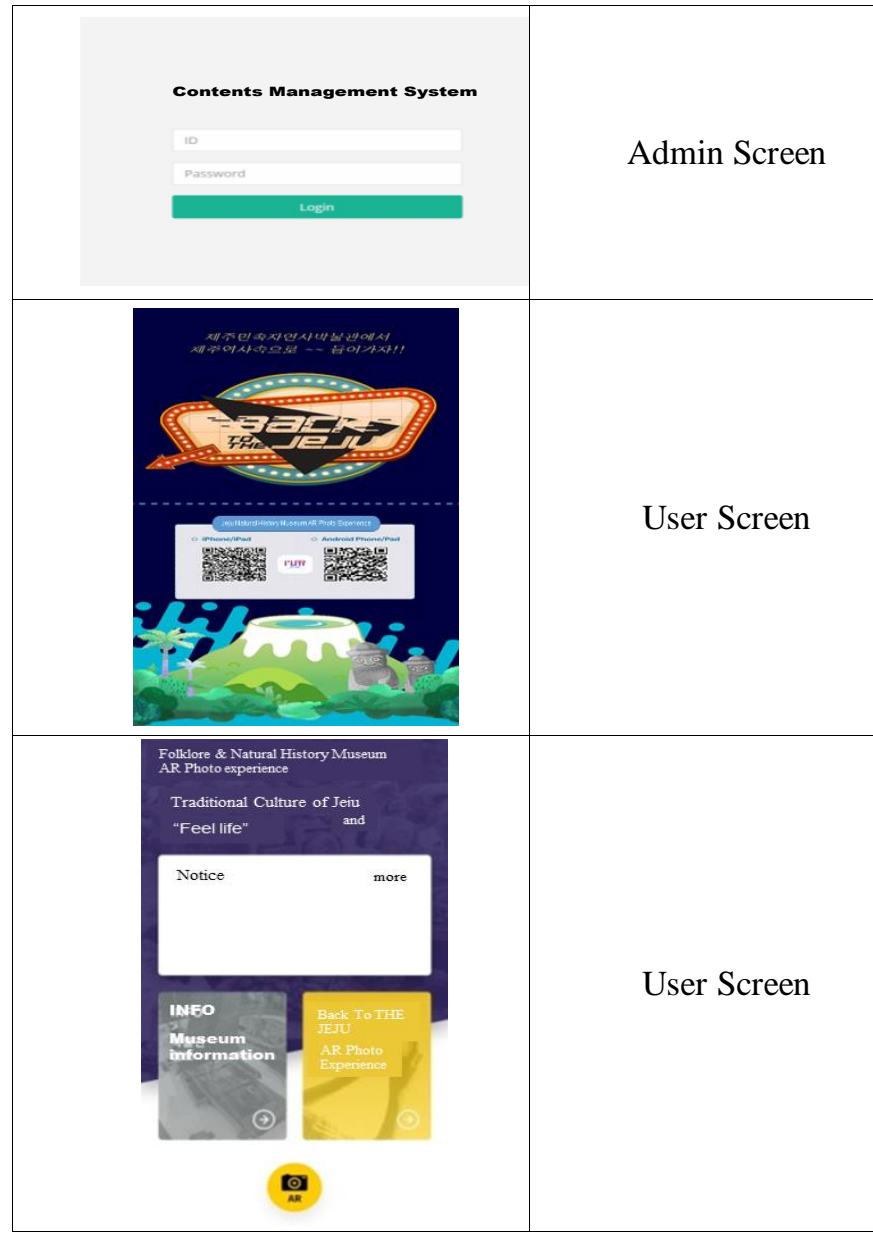

$<$ Figure 9> Login screen

3.3.2Kiosk display screen

A self-shot was taken in front of a kiosk in the exhibition hall (phone number entered). The application was then downloaded, which showed the works of the exhibition hall (display markers recognized). The image taken in the self-shot will be provided in the application, and the composite image of the displayed work or AR and video contents will be provided as well.

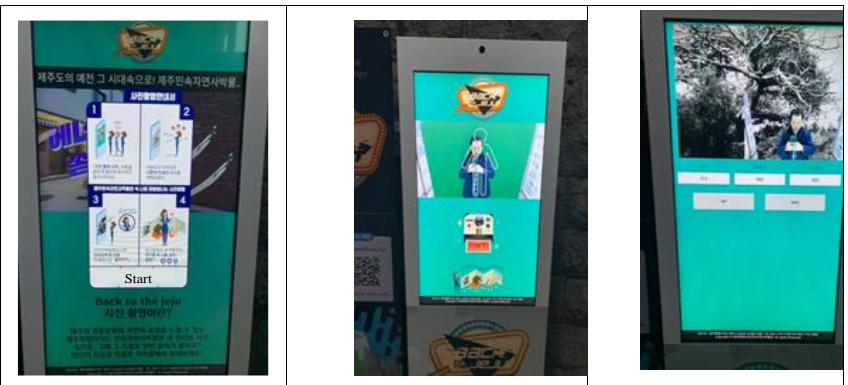

$<$ Figure 10> Kiosk implementation and the interlinking module screen 
3.3.3 Implementation of the transparent screen

Content is displayed on transparent screens installed in exhibition halls. Devices are selected at the transparent screens through the integrated CMS and registered as new content (a promotional video), then checked to see if it is provided normally at the transparent screen

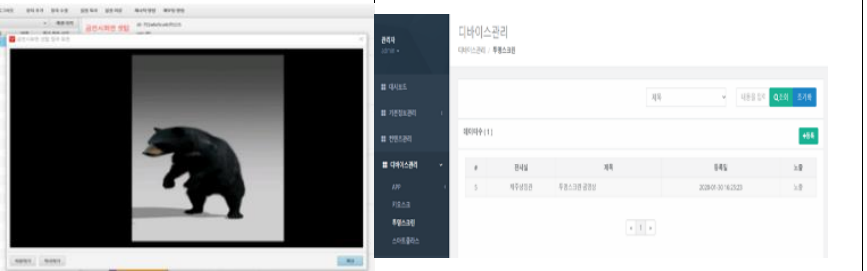

<Figure 11> Transparent screen implementation and the interlinking module screen

3.3.4 Smart glasses / application implementation screen

If smart glasses are worn in front of a Teu exhibition in the exhibition hall, markers will be recognized through the smart glasses. The Teu content in the exhibition will be provided to the smart glasses as shown below.

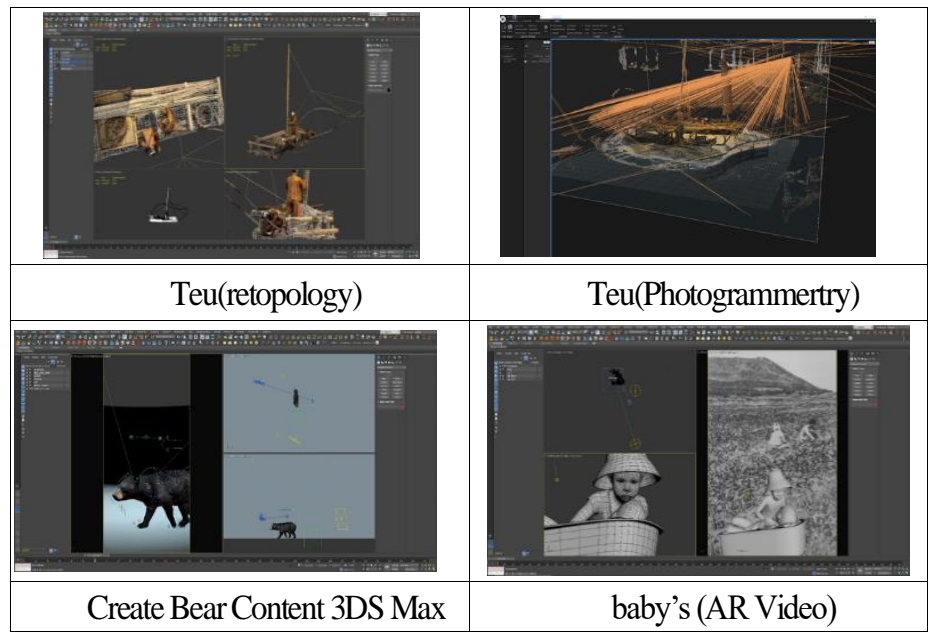

$<$ Figure 12> Implementation screen in smart glasses / application

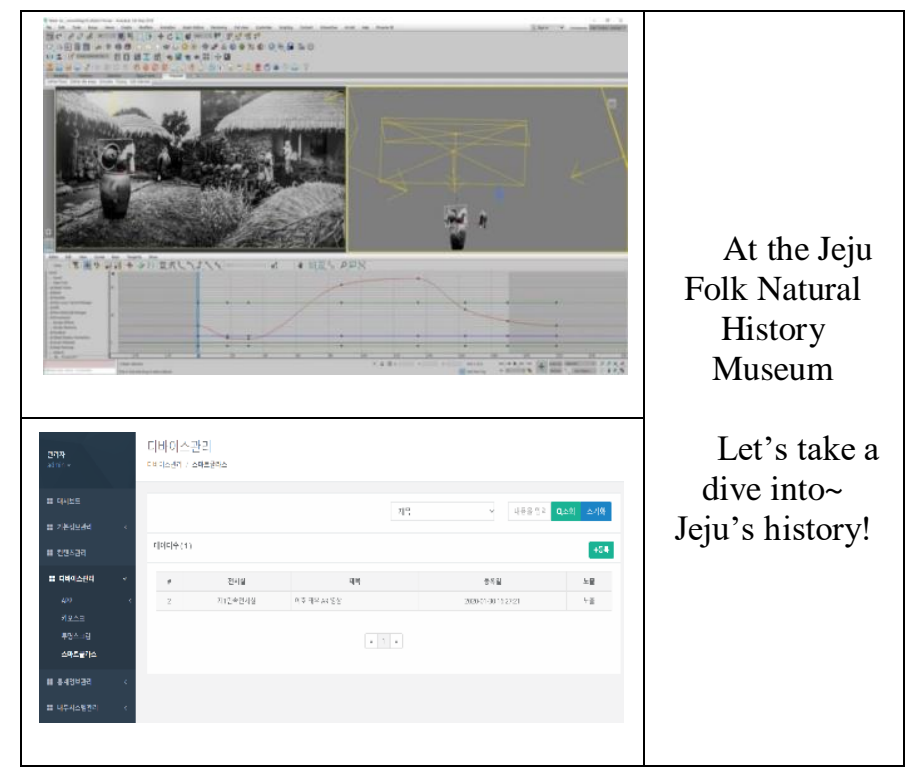

$<$ Figure 13> Interlinking module screen in smart glasses/ application

This service consists of both a user-level and an administrator-level service. 


\section{Conclusions}

In this study, a realistic smart information guide solution was developed through support for multi-devices. This system enabled integrated contents and operation management concurrent with the support of smart devices with different, specific characteristics. Through the development of the realistic smart information guide system, it is possible to install realistic content and support smart devices while also improving the operation management of integrated content and reducing the cost of such management.

Along with the development of an integrated solution for realistic smart information guidance that supports multiple devices, the quality of contents (AR, VR) used in the solution can be important. It can be seen that it is an efficient solution for operations and administrators, but it can be more effective as an answer for museums and exhibition halls to allow them to interact more with the contents being provided to increase viewer involvement. With this point of view on the matter, it will be necessary to actively develop AR and VR content in the future and to plan practical content services for visitors and tourists for locations such as museums and exhibition halls.

\section{References}

1. Kim Sung.Hee., Lee Hyoung.Guk., RohHyun.Suk., \& LeeHyun.Woo.(2015). Advanced Technologies for Smart Exhibition Guide Service. Electronic and Telecommunications Trends. 30(3), 42-51.

2. Kim Young-Ick., Kim Mi-jung., \& Kim Hyu-Chan.(2016). Asia-pacific Journal of Multimedia Services Convergent with Art.Humanities, and Sociology, 6(2), 35-44.

3. http://isilab.kaist.ac.kr/?p=4800, 2018

4. http://www.doopedia.co.kr

5. Im Yang-Mi.(2016). Virtual Reality Content and Technology Trends. Information \&communications magazine . 33(12). 52

6. Kim Kyoung-Sub., Park Young-Cheol., Eom Chan-jin., Lee Yun-Bok.,\& Lee Sang-Ho.(2019). Implementation of a Location-Based SNS System Using AR.The Journal of The Institute of Internet, Broadcasting and Communication(IIBC), 19(6), 133-138.

7. Im sang-woo, seoKyoung-Won.(2018). AR/VR Technical, KISTEP, 9, 2.

8. Lee Sang-Hyun(2017). A Study on Development of Experimental Contents Using 3-channelMultiImage Playback Technique: Based on transparent OLED and duallayer display system.The Journal of The Institute of Internet, Broadcasting and Communication(IIBC),17(6), 152.

9. Park Jong-man, JwangJea-ryoung, Kim Ha-jin (2013). The Technical Compositions, Trends and Prospects.Communications of the Korean Institute of Information Scientists and Engineers, 31(9), 53-60

10. Kim Hang-kyu.(2019). Progression of Business Trends inAugmented Reality Devices, Monthly software oriented society, 9-10

11. David, O. O., \& Grobler, W. (2019). Agricultural Production In South Africa: Information And Communication Technology (Ict) Spillover. International Journal OfEbusiness And Egovernment Studies, 11(2), 167-190.

12. Erol, İ., \&Velioğlu, M. N. (2019). An Investigation Into Sustainable Supply Chain Management Practices In A Developing Country. International Journal OfEbusiness And Egovernment Studies, 11(2), 104-118. 